\title{
State-Dependent Riccati Equation Control with Predicted Trajectory
}

\author{
A. S. Dutka and M. J. Grimble \\ Industrial Control Centre, University of Strathclyde, Glasgow, UK
}

\begin{abstract}
A modified State-Dependent Riccati Equation method is used which takes into account future variations in the system model dynamics. The system in the state dependent coefficient form, together with the prediction of the future trajectory, may be considered to be approximated by known time-varying system. For such a system the optimal control solution may be obtained for a discrete time system by solving the Riccati Difference Equation. The minimisation of the cost function for a predicted time-varying system is achieved by considering the prediction horizon as a combination of infinite and finite horizon parts. The infinite part is minimised by solving the Algebraic Riccati Equation and the finite part by the Riccati Difference Equation. The number of future prediction steps depends upon the problem and is a fixed variable chosen during the controller design. A comparison of results is provided with other design methods, which indicates that there is considerable potential for the technique.
\end{abstract}

\section{INTRODUCTION}

There is a need for control laws that are simple to compute, suitable for nonlinear systems [1] that may be optimised in some sense [2]. The family of LQ and LQG design methods $[3,4]$ have been very successful for linear systems and the aim is to provide an equally simple method that can be used for nonlinear systems. Linear quadratic optimal control [5] results, for time-varying linear systems are used. The main idea is to estimate the future variations in the nonlinear system characteristics [6] and to then apply the linear time-varying optimal control results. The class of systems being considered are those which can be approximated by a state-space model with time dependent parameters.

A restricted class of nonlinear systems is used, which is the same as that employed in papers on the "StateDependent Riccati Equation" approach [7-12]. That is, the nonlinear system has a form that is like a linear state-space description but where the system matrices are functions of state. In the so called State-Dependent Riccati Equation method the calculations are performed, assuming the system remains fixed (time-invariant) at the values for the current operating condition. The linear system matrices calculated at this point are then used for the solution of Algebraic Riccati Equation. The State-Dependent Riccati Equation technique assumes that the system may be approximated using the linear time-varying system model, since the State Dependent model has a linear structure and the system matrices depend on the state, which is assumed to be available at the current time instant $k$.

It was reported [13] that the state-dependent Riccati equation method has many advantages over other nonlinear design methods. The main drawback is the lack of a guarantee of global asymptotic stability which in general is a difficult issue for non-linear systems. The local stability at the origin of the closed loop system results from the stabilising properties of the solution of the Algebraic Riccati Equation. Unfortunately, so far, one of the most efficient methods of assessing the stability of the SDRE controller is by simulation. Recent work in the stability analysis of the SDRE method either gave rather difficult conditions to check or imposed difficult requirements. In [14] the region of attraction for the SDRE controller, around the origin of the closed loop, is determined and for this region the stability of the controller is guaranteed. This may be difficult since closed-loop system equations are usually not known explicitly. In [15] the stability of the system controlled by the SDRE method is ensured via "satisficing" provided that a Control Lyapunov Function for considered system is known. The main difficulty with this technique is to find the global Control Lyapunov Function for the non-linear system. For some systems such a function may easily be determined and in this case the method may be employed. In [16] the estimation of the region of stability is substituted by the functional search problem. The State-Dependent model matrices were assumed to be polynomial functions of the state and the stability region estimate was obtained though optimisation. In the SDRE method where the Algebraic Riccati Equation is solved, using state-space matrices calculated at the current state it is assumed implicitly that the system in the future will remain fixed at the current operating point which is equivalent to the assumption that the system is time invariant with the system model fixed at the current time instant. This assumption represents a severe approximation since this is true only for the origin. Therefore there is only a guarantee of local stability for SDRE and as stated in $[14,16]$ some region of attraction around the origin may be determined (this region may ideally cover the whole operating range).

In this paper it is assumed that prediction of the future state trajectory may be determined. With this knowledge the Algebraic Riccati Equation may be solved not just for the current state (as it was done in the SDRE) but for the prediction of the future state. For a discrete time system controlled at time $k$ it would mean that the ARE is solved at 
$k+k_{p}$, where $k_{p}$ is the last prediction available. If the state at $k_{p}$ time instant represents the steady state of the system then the solution of ARE may be used as a boundary condition for the solution of the Difference Riccati Equation which is iterated backwards using available predictions of the system matrices. Finally the state feedback gain and the control signal may be obtained.

The assumption on the knowledge of the state trajectory may be satisfied at a given time instant $k$ first estimating the current and future control signal values for $k, k+1, k+$ $2, \ldots, k+k_{p}-1$. These values might for example be approximated using the last calculated value of the gain matrix $K_{C}(k-1)$ and the State-Dependent model of the system. Alternatively, an estimate of the future control can be computed assuming that the system parameters will remain fixed at their current values. Using the model and the control signal estimates the prediction of the state trajectory may be determined. This provides an indication of the likely time variation of the system matrices. Given the time-varying system matrices the linear time-varying quadratic optimal controller results may then be applied. Thus the solution of the Algebraic Riccati Equation is first determined using the system model at time $k+n_{p}$, which is assumed time invariant from that point on. The solution of the Algebraic Riccati Equation (say $P_{\infty}$ ) can then be used to initialize the time-varying Riccati difference equation to solve (backwards in time) for $P(k+1)$. The values of the Riccati solution $\{P()$.$\} at times k+k_{p^{-1}}, t+k_{p^{-2}}, \ldots, t+1$ may then be computed. The gain at time $k$, which is to be used to compute the control signal at time instant $k$ then follows. The whole process must be repeated at the next time instant.

\section{SYSTEM DESCRIPTION}

The system is assumed to be approximated by a time-varying linear system with a state-space structure. This structure results directly from the state-dependent form of state-space system. The limited class of non-linear systems of interest are those which can be modelled by such state-space model $[9,10]$. Let the general (underlying) non-linear state-space model be given by the following equation:

$$
\begin{aligned}
& x_{p}(k+1)=f\left(x_{p}(k)\right)+B_{p}\left(x_{p}(k)\right) u_{p}(k) \\
& y_{p}(k)=C_{p}\left(x_{p}(k)\right) x_{p}(k)
\end{aligned}
$$

The assumption is now made that the function $f\left(x_{p}(k)\right)$ may be re-written in the form: $A_{p}\left(x_{p}(k)\right) x_{p}(k)$. Detailed discussion of the possible methods of getting the statedependent form is given in [17]. In general there is an infinite number of such re-arrangements. This may be regarded as an additional degree of design freedom. To obtain a solution of the Algebraic Riccati Equation the assumption that for all $x_{p}$ the pair $\left(A_{p}\left(x_{p}\right), B_{p}\left(x_{p}\right)\right)$ is point-wise controllable must be made. This assumption may be relaxed to stabilizability of the given pair with an additional requirement on observability through state weighting matrix. As a slight additional generalisation the state matrices may also be assumed control signal dependent.

As outlined in the introduction the prediction of state trajectory is used $x_{p}(k+1) \ldots x_{p}\left(k+k_{p}\right)$ to calculate the prediction of the future system model matrices. Thus, for some time into the future the system can be approximated by a known time-varying linear state-space model.

To simplify notation $A_{p}\left(x_{p}(k)\right), B_{p}\left(x_{p}(k)\right)$, $C_{p}\left(x_{p}(k)\right)$ are denoted as $A_{p}(k), B_{p}(k), C_{p}(k)$.

\section{Plant model:}

$x_{p}(k+1)=A_{p}(k) x_{p}(k)+B_{p}(k) u_{p}(k)$

$y_{p}(k)=C_{p}(k) x_{p}(k)$

The model will include the following reference signal model:

Reference signal model:

$x_{r}(k+1)=A_{r} x_{r}(k)$

$y_{r}(k)=C_{r} x_{r}(k)$

Combining these equations the total augmented system, whose states are assumed to be available for feedback, become:

\section{Augmented System}

$x(k+1)=A(k) x(k)+B(k) u(k)$

$y(k)=C(k) x(k)$

The augmented system matrices are defined as:

$$
\begin{aligned}
A(k) & =\left[\begin{array}{cc}
A_{p}(k) & 0 \\
0 & A_{r}
\end{array}\right], \quad B(k)=\left[\begin{array}{c}
A_{p}(k) \\
0
\end{array}\right], \\
C(k) & =\left[\begin{array}{ll}
C_{p}(k) & 0
\end{array}\right] .
\end{aligned}
$$

\section{CONTROL ALGORITHM}

The way in which the accuracy of the solution of the SDRE can be improved will now be considered. 
Assuming that it is possible to determine the future trajectory of the system with a certain accuracy the nonlinear system may be approximated using linear timevarying model. With this knowledge the evident drawback of the SDRE assumption of the system invariance from the current time into the future may be removed if the future trajectory was known from the initial state to the origin. In this situation the state-dependent model can be replaced by the known time-varying linear system and the solution of this problem is straightforward. In practice it is not possible to obtain the state trajectory, only the prediction with a certain accuracy may be determined.

The approximate prediction of the state trajectory may be calculated using the state feedback gain $K_{c}(k-1)$, obtained in the previous iteration of the control algorithm. It may be used to calculate the approximate control action for the current time instant. Given the current state measurement (or estimate) the state-space model matrices at the current time instant are obtained and the future state prediction may be calculated. Using the same state feedback gain (from the previous iteration of the control algorithm) a prediction of the next (future) control action may then be obtained. This procedure can be repeated and finally future states $x_{p}(k+1) \ldots x_{p}\left(k+k_{p}\right)$ can be obtained and used for calculation of the future system model matrices.

The specification of the control algorithm may now be outlined. The cost-index involves the minimisation of the quadratic cost function [19] including the state and control:

$$
J_{k}=\sum_{n=k}^{k+\infty}\left\{x^{T}(n) Q(n) x(n)+u^{T}(n) R(n) u(n)\right\}
$$

where

$$
Q(n)=\left[\begin{array}{ll}
C_{p}(n) & -C_{r}
\end{array}\right]^{T} \bar{Q}\left[\begin{array}{ll}
C_{p}(n) & -C_{r}
\end{array}\right]
$$

and the weightings : $\bar{Q} \geq 0$ and $R>0$.

The weighting matrices $\bar{Q}$ and $R$ may depend on the state of the system and the resulting cost function may not in general therefore be quadratic.

The minimization of the cost function subject to the non-linear system dynamics requires a solution of the non-linear optimization problem that in general difficult to obtain. To avoid this problem the minimisation of the cost function is performed subject to the linear approximation of the non-linear system. For a non-linear system the future values of the approximate linear system matrices for the infinite horizon are not really available. In the State Dependent Riccati Equation method the cost function is minimised with the assumption that system will remain time invariant from the current time on. In this case the cost-function is measured over the interval $k$ to infinity.
This corresponds to the interval over which the timevarying model must be assumed to be known, if the usual optimal control solution for linear systems is to be applied. The way round this problem is to assume the state and control action up to say time $k+k_{p}-1$ is calculable, so that the system is known up to $k+k_{p}$. The length of the prediction horizon may be regarded as a tuning parameter. The prediction of the future trajectory is likely to be mismatched for longer horizons. The system's non-linearity will have a significant impact on the prediction accuracy. For highly non-linear systems it may be necessary to reduce the horizon due to precision limitations. Also, weights in the cost function should be taken into consideration when the length of the horizon is chosen. A higher control penalty will result in slower response of the closed loop system. Consequently, the system state variation will be slower and the accuracy of the prediction within given horizon improved. After the time $k+k_{p}$ the system matrices will be assumed to remain constant. Thus, a control action may be computed at time $k$ and for future times. In a similar way at time $k+1$ the system is assumed known up to $k+k_{p}+1$ and is constant thereafter. Thus, the new control signal is computed at $k+1$ should be implemented, which is in the spirit of a receding horizon philosophy.

$$
\text { Assuming that after the } k_{\infty, k}=k+k_{p} \text { time instant }
$$

the system will remain fixed then the cost function may be re-written into the following form:

$$
\begin{aligned}
& J_{k}=J_{k}^{\text {finite }}+J_{k}^{\text {infinite }} \\
& J_{k}^{\text {finite }}=\sum_{n=k}^{k+k_{p}-1}\left\{x^{T}(n) Q(n) x(n)+u^{T}(n) R(n) u(n)\right\} \\
& J_{k}^{\text {infinite }}=\sum_{n=k+k_{p}}^{k+\infty}\left\{\begin{array}{l}
x^{T}\left(k_{\infty, k}\right) Q\left(k_{\infty, k}\right) x\left(k_{\infty, k}\right) \\
+u^{T}\left(k_{\infty, k}\right) R\left(k_{\infty, k}\right) u\left(k_{\infty, k}\right)
\end{array}\right\}
\end{aligned}
$$

The minimisation of the second part - $J_{k}^{\text {infinite }}$ is obtained easily from an Algebraic Riccati Equation, calculated at time $k+k_{p}$. The solution of the Algebraic Riccati Equation does of course minimize the cost assuming the system is time-invariant. This solution is applicable since it is assumed, that the system will remain fixed after time $k+k_{p}$. The Algebraic Riccati Equation is given by the following expression calculated at the time instant $k_{\infty, k}=k+k_{p}$ : 


$$
\begin{aligned}
& P_{\infty}\left(k_{\infty, k}\right)=A\left(k_{\infty, k}\right)^{T} \times \\
& {\left[\begin{array}{c}
P_{\infty}\left(k_{\infty, k}\right)-P_{\infty}\left(k_{\infty, k}\right) B\left(k_{\infty, k}\right)^{T} \times \\
\left(R\left(k_{\infty, k}\right)+B\left(k_{\infty, k}\right)^{T} P_{\infty}\left(k_{\infty, k}\right) B\left(k_{\infty, k}\right)\right)^{-1} \times \\
B\left(k_{\infty, k}\right) P_{\infty}\left(k_{\infty, k}\right)
\end{array}\right]} \\
& \times A\left(k_{\infty, k}\right)+Q\left(k_{\infty, k}\right)
\end{aligned}
$$

After computing the control to minimize the cost term from $k+k_{p}$ onwards the next step is to minimise the first part of the cost function so that the $J_{k}^{\text {finite }}$ is minimised. This minimisation problem involves the finite time cost function term. The solution of the Algebraic Riccati Equation is taken as a boundary condition $P\left(n+n_{p}\right)=P_{\infty}\left(k_{\infty, k}\right)$ for the Riccati difference equation:

$$
\begin{aligned}
& P(n)=A(n)^{T} \times \\
& {\left[\begin{array}{l}
P(n+1)-P(n+1) B(n)^{T} \times \\
\left(R(n)+B(n)^{T} P(n+1) B(n)\right)^{-1} \times \\
B(n) P(n+1)
\end{array}\right] A(n)}
\end{aligned}
$$

$$
+Q(n)
$$

The Riccati difference equation iterations are performed for $n=\left(k+k_{p}-1\right), \ldots,(k+1)$. Finally the control signal at time $k$ is obtained from the discrete time-varying Kalman gain expression, assuming the states are available for feedback, as:

$$
u(k)=K_{c}(k) x(k)
$$

where

$$
\begin{aligned}
K_{c}(k)= & -\left(B(k)^{T} P(k+1) B(k)+R(k)\right)^{-1} \\
& \times B(k)^{T} P(k+1) A(k)
\end{aligned}
$$

The stability issue may be tackled in a similar way as for the SDRE. As was noted in the introduction stability analysis for the nonlinear systems controlled by the SDRE algorithm may be difficult. In general it is not possible to have an explicit equation of the closed loop system. Hence for stability analysis the only suitable method seems to be that given in [15]. If the Control Lyapunov Function is known the method may be easily implemented and as a result a guarantee of global asymptotic stability achieved. The weakness of this method is the difficult issue of generating a suitable CLF for the non-linear system. If this is difficult then the only method to check the stability properties for the given application is to use simulation.

\section{SUMMARY OF THE ALGORITHM}

There follows a list of the main steps in the computational algorithm:

1. Estimate (or measure) the state

2. Use a previous feedback gain to calculate the prediction of the current control

3 . Use the current control prediction and the model recalculated at time instant $k$ to obtain the future state prediction. The state prediction together with the state feedback gain from previous iteration of the algorithm is used for the calculation of the future control prediction. The model once again is re-calculated using future state prediction, stored and the sequence can be repeated $k_{p}$ times.

4. Use the model prediction for time instant $k+k_{p}$ and solve Algebraic Riccati Equation.

5. Use $P_{\infty}\left(k_{\infty, k}\right)$ as a boundary condition $P\left(k+k_{p}\right)$ for iterations of the Difference Riccati Equation and then use an appropriate prediction of the model through iteration of Riccati Equation.

6. Use $P(k+1)$ to calculate the feedback control gain and calculate the current control.

\section{NONLINEAR SERVO-SYSTEM EXAMPLE}

To illustrate the potential possibilities of the proposed algorithm a simple second order non-linear unstable system is going to be controlled. The proposed algorithm is compared with SDRE method and with a linear controller. The block diagram of the object is shown in Fig.3.

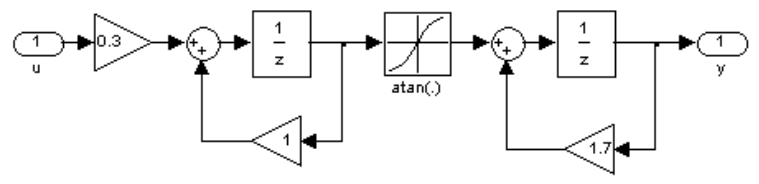

Figure 1: Block Diagram.

The system is non-linear and the open loop unstable (pole out of the unit circle) and the state space model is given by the following equations:

\section{Plant model:}

$x_{p}(k+1)=\left[\begin{array}{cc}1.7 & \frac{\operatorname{atan}\left(x_{p, 2}(k)\right)}{x_{p, 2}(k)} \\ 0 & 1\end{array}\right] x_{p}(k)+\left[\begin{array}{c}0 \\ 0.3\end{array}\right] u(k)$ 


$$
y_{p}(t)=\left[\begin{array}{ll}
1 & 0
\end{array}\right] x_{p}(t)
$$

with initial condition $x_{r}(0)=$ setpoint. In the example setpoint $=1.2$ and was chosen such, that the plant works on the non-linear part of the saturation characteristic.

Reference Model:

$x_{r}(t+1)=[1] x_{r}(t)$

$y_{r}(t)=[1] x_{r}(t)$

with initial condition $x_{r}(0)=$ setpoint. In the example setpoint $=1.2$ and was chosen such, that the plant works on the non-linear part of the saturation characteristic.

The step response of the closed loop system is shown in Figure 2 and the corresponding control action is shown in figure 3 .

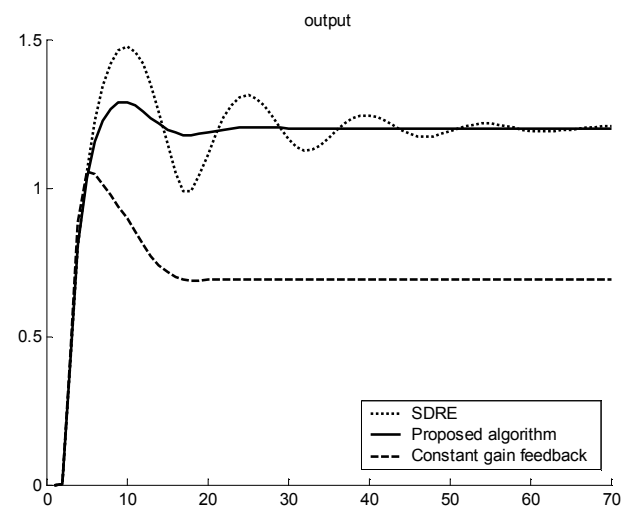

Figure 2: Output Responses for Comparison

In the example it was assumed that states were available for measurement. However with the model given in the form shown in figure 2 it is straightforward to implement a Kalman Filter.

From analysis of the output response it may be concluded that the linear controller gave a significant steady-state error. The constant gain used for state feedback was designed for the plant parameters calculated around its parameters corresponding to the setpoint value. When the gain was designed using the initial system parameters, the system with such a controller did not give a stable response.

It may be noted that the proposed algorithm gives significantly better performance, compared to a SDRE controller and this gives better performance when compared to a linear controller. The latter stabilises the system, but the response, especially in terms of the steady state error, is not adequate.

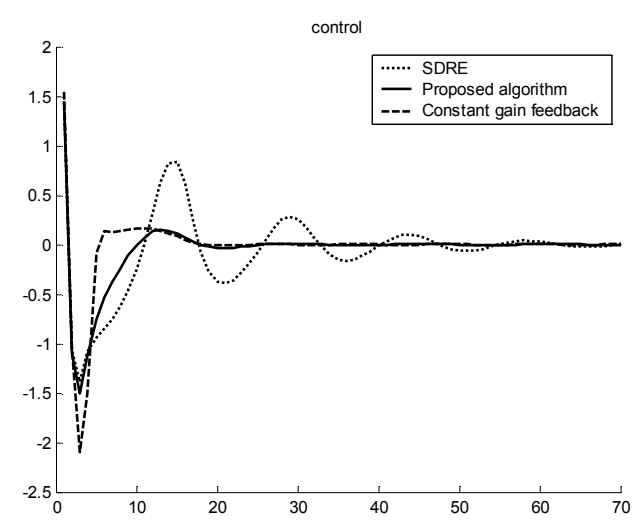

Figure 3: Control Signals for Comparison

\section{CONCLUDING REMARKS}

The main advantage of the proposed technique is the simplicity of the approach. In the steady-state the control law reduces to the optimal control for a timeinvariant system, which for small perturbations is desirable. When there are large reference or disturbance signal changes the control law is evaluated taking into account the future changes in the system parameters brought about by the presence of non-linearities. This is an improvement over the state-dependent Riccati equation method, which assumes the system remains fixed at the nonlinear function values at the time $t$. A comparison of the results for the example reveals that valuable improvements are obtained, even for a relatively small number of steps $k_{p}$.

For most nonlinear control design approaches stability issues are central to the theory and this requires either elegant mathematical results or empirical procedures [18]. The approach above is optimisation based and the focus is more on the performance, under different operating conditions. The analysis of performance is rather easier to achieve, either from operating records, or from theoretical results. Thus, the confidence necessary to encourage the use of the approach is more likely to be achieved by this optimization method. This does not imply that a measure of stability is not important, but it changes the focus of the design onto property, which is easier to measure and benchmark.

\section{ACKNOWLEDGEMENTS}

We are grateful for the support of the Engineering and Physical Sciences Research Council on the Nonlinear Control Project, Platform Grant GR/R04683/01. Discussions with Dr. Andrzej Ordys of the University of Strathclyde are gratefully acknowledged. 


\section{REFERENCES}

1. Bendat, J. S., 1998, Nonlinear System Techniques and Applications, (Wiley-Interscience, New York)

2. Isidori, A., 1995, Non-linear control systems, (Springer Verlag, Berlin, $3^{\text {rd }}$ Edition)

3. Kwakernaak, H. and Sivan, R., 1972, Linear optimal control systems, (John Wiley)

4. Grimble, M. J. and Johnson, M. A., 1988, Optimal control and stochastic estimation (Vols I and II, John Wiley, Chichester)

5. Anderson, B., and Moore, J., 1971, Linear optimal control, (Prentice Hall, Englewood Cliffs)

6. Mohler, R. R., 1991, Nonlinear Systems, (Vol. 1, Dynamics and Control, Prentice Hall)

7. J. D. Pearson, 1962, Approximation Methods in Optimal Control, Journal of Electronics and Control, 13, 453-465

8. J. H. Burghart, 1969, A Technique for Suboptimal Feedback Control of Nonlinear Systems, IEEE Transactions on Automatic Control, 14 530-533

9. J.R Cloutier, C.N. D'Souza and C.P. Mracek, 1996, Nonlinear regulation and Nonlinear H-infinity Control via the State-Dependent Riccati Equation Technique: Part 1, Theory, Part 2, Examples, Proceedings of the 1st International Conference on Nonlinear Problems in Aviation and Aerospace, 117-141, 1996

10. J. R. Cloutier, 1997, State-Dependent Riccati Equation Techniques: An Overview, Proceedings of the American Control Conference, 2, 932-936,

11. Y. Huang, W-M. Lu, 1996, Nonlinear Optimal Control: Alternatives to Hamilton-Jacobi Equation, Proceedings of the 35th IEEE Conference on Decision and Control, 3942-3947,.

12. Hammett, K. D., 1997, Control of Non-linear systems via state-feedback state-dependent Riccati equation techniques, $\mathrm{PhD}$ Dissertation, Air Force Institute of Technology, Dayton, Ohio,

13. D’Angelo, H., 1970, Linear time-varying systems: analysis and synthesis, Allyn and Bacon, Boston

14. Erdem, E. B., Alleyne, A. G., 2002, Estimation of Stability Regions of SDRE Controlled Systems Using Vector Norms, Proceedings of the American Control Conference, Anchorage 2002, 80-85

15. Curtis, J. W., Beard, R. W., Ensuring Stability of Statedependent Riccati Equation Controllers Via Satisficing, Proceedings of the 41st IEEE Conference on Decision and Control, 2645-2650

16. Seiler, P., Stability Region Estimates for SDRE Controlled Systems Using Sum of Squares Optimization, Proceedings of the American Control Conference, Denver 2003, 1867-1872

17. Cloutier, J. R., Stansbery, D. T., The Capabilities and Art of State-Dependent Riccati Equation-Based Design, Proceedings of the American Control Conference, Anchorage 2002, 86-91
18. Atherton, D. P., 1975, Non-linear Control Engineering, ((London, Van Nostrand Reinhold)

19. Grimble, M. J., 2001, Industrial Control Systems Design, (John Wiley, Chichester) 